\title{
SOLID-LIQUID FLOW OF AXIAL FLOW PUMP IN LOOP REACTOR AND OPERATING CONTROL WITH SINGLE INVERT
}

\author{
Yan, H. ; Wang, Y. R.., ; Shi, H. X. ; Li, Q. ; Zeng, Y. S. ${ }^{*} \&$ Jaini, R. ${ }^{* * *}$ \\ *School of Mechanical Engineering, Hefei University of Technology, 193 Tunxi Road, Hefei City, \\ 230009, Anhui, China \\ ${ }^{* *}$ School of Mechanical Engineering, Wanjiang University of Technology, 333 Huolishan Avenue, \\ Ma'anshan City, 243000, Anhui, China \\ ${ }^{* * *}$ Research and Production Centre, Empire Industrial Equipment Ltd., M. G. Road IFFCO Chowk, \\ Gurugram, Haryana, 122002, India \\ E-Mail: wangyr1210@126.com
}

\begin{abstract}
Solid concentration changes may affect the solid-liquid flow of the axial flow pump in the polypropylene loop reactor, accompanied with unstable operations (e.g., axial power fluctuation). The influencing factors and formation mechanisms of axial power fluctuation were analysed. After that, to discuss the influences of energy allowance of the mixture media in the loop reactor, the internal flow status of the pump under different solid volume fractions were compared. Then a head-correction strategy of operating control with single invert was proposed, to smooth the axial power fluctuation of the axial flow pump. Results demonstrate that viscosity of the mixture media is positively related, whereas the head of the axial flow pump is negatively correlated with solid concentration. Besides, head-correction curves can effectively regulate the off-design operation of pump under different solid volume fractions, with the energy allowance and the axial power fluctuation reduced in the loop reactor and the pump. Researches provide important references for the analysis of internal flow status and the solution of axial power fluctuation of the axial flow pump in the polypropylene loop reactor. (Received in April 2019, accepted in August 2019. This paper was with the authors 2 months for 1 revision.)
\end{abstract}

Key Words: Axial Flow Pump, Solid-Liquid Flow, Axial Power Fluctuation, Operating Control with Single Invert

\section{INTRODUCTION}

Axial flow pump is a cycle driving device in a polypropylene loop reactor. The loop reactor is a common synthesis device of polypropylene. In the reactor, propylene produces polypropylene particles under the effect of catalyst with particles suspended in liquid, thereby forming the mixture dominated by liquid (propylene) and solid (polypropylene) phases. Nevertheless, the existence of solid particles may affect the operating performance of the pump [1]. The solid-liquid flows of the axial flow pump in the loop reactor must be investigated to realize the high-efficiency performance of the pump. In addition, the axial flow pump is designed on the basis of water media, which differ from the state of practical flowing medium in the reactor [2]. Therefore, the performance changes of axial flow pump must be analysed under different media and running conditions and changing working conditions.

Existing studies on loop reactor focus on the mathematical modelling of residence time distribution [3]. The characteristics of computational fluid dynamics (CFD) on internal solidliquid flow states in loop reactors, especially the core device in a loop reactor, namely, axial flow pump, are hardly analysed. Additionally, influences of solid volume fraction in the solidliquid mixture in the reactor on liquid properties of the mixture are hardly considered. For mixing fluid containing solid particles, fluid properties may change when the solid volume 
fraction increases to a certain numerical value, that is, the Newtonian fluid is converted into Bingham fluid (non-Newtonian fluid). As a result, CFD analysis will make changes [4]. Consequently, studies on solid-liquid flows focus on the low concentration range. Moreover, research concerning the operation instabilities of axial flow pump, such as axial power fluctuation, mainly emphasizes fault diagnosis and engineering solutions. No theoretical solution to the formation mechanism of operation instabilities has been developed at present. In this study, key attention is given to disclose the variation law of internal flow of the axial flow pump in the loop reactor under different media conditions and the internal relationship with axial power fluctuation. Reasonable solutions are subsequently proposed.

\section{STATE OF THE ART}

Numerous studies on two-phase flow have recently been reported. However, the two-phase flow has become an independent discipline after the International Journal of Multiphase Flow was founded in the 1960s. Based on different dispersed phase hypotheses, numerical computation methods of two-phase flows mainly include Eulerian-Eulerian and EulerianLagrangian methods [5]. The Eulerian-Eulerian method simplifies the dispersion phase into a pseudo-fluid, wherein continuous phases penetrate mutually. The essence of this method is to simulate the turbulence of dispersion phases imitated by a single-phase flow $[6,7]$. The Eulerian-Lagrangian method describes the dispersion phase as a discrete body in the Lagrangian coordination system and presents the continuous phase in the Eulerian coordination system $[8,9]$. Most numerical calculations of solid-liquid flows in the pump have applied the mixture model, which hypothesizes both phases as continuous media [1]. Nevertheless, the less-known particle model, which hypothesizes one phase as continuous and the other phase as dispersion, has relatively high calculation accuracy [10].

Zhao et al. [1] conducted a numerical simulation of solid-liquid flows in a two-channel pump on the basis of FLUENT software by using the mixture multiphase model. Influences of different particle concentrations and sizes on the external characteristics of the pump and the solid-phase distribution laws were analysed. However, the mixture model was extensively applied to multiple groups of continuous media mixture, such as gas-liquid. The numerically calculated head of solid-liquid two-phase flow significantly deviated from the test results. Shi and Wei [11] increased the astringency of solid-liquid two-phase flow numerical calculation in a centrifugal slurry pump with low specific speed by combining the dynamic under relaxation factor adjustment, step method, and rotational velocity control. Shi et al. [12] designed a new no-stirring test device for the PIV test of solid-liquid two-phase flows in the same pump. They found that the speed distribution law on test images of the two-phase flow based on water and rapeseed conforms to potential flow theory. However, Shi analysed different concentrations of media by using the same analytical method but ignored the property changes of high-concentration fluid, resulting in lack of rigor. Zhang et al. [13] investigated the influences of solid-liquid flow in the transient operational processes (e.g., starting and stopping) of a centrifugal pump on the basis of FLUENT software. They concluded that the pump pressure in starting and stopping processes under solid-liquid flows fluctuates further violently, the axial reactive force of the impeller increased, and the time taken to stabilize increased. Shi et al. [14] performed a numerical calculation on solid-liquid two-phase flows in an axial flow pump based on ANSYS CFX software by using the particle model. They analysed influences of impellers with different sweepbacks on the cavitation performance and abrasion of the pump. However, the $30 \%$ solid concentration was insufficient to provide references compared with sediment contents in practical operation conditions. Based on the Eulerian-Lagrangian method, Lei et al. [15] analysed the influences of abrasion degree on the external properties of a centrifugal pump by using the SST $k-\omega$ 
turbulence model. They found that with the increase in abrasion degree, the head and efficiency of the pump increase under small flow speeds, but decrease under large flow speeds. Numerous studies on solid-liquid two-phase flows in a loop reactor have been reported. Based on the Eulerian-Eulerian method, Shi et al. [16] analysed the effects of circulating flow speed in the pipeline and solid particle diameter on solid-phase retention when the solid volume fraction is 0.35 . Yan et al. [17] studied the influences of guide vane on solid-liquid two-phase flows in a loop reactor under the same conditions by using the same physical model and analysis method as Shi [16]. Li et al. [18] analysed the fluctuations of speed, pressure, and solid volume fraction based on the transient Eulerian-Eulerian model when the solid volume fraction is 0.23 . However, the mixture of high-concentration solidphase polypropylene and liquid-phase propylene could not maintain the physical properties of Newtonian fluid, but showed properties of Bingham fluid. The Eulerian-Eulerian model was only applicable to low-concentration solid-liquid two-phase flows.

Operating control with single invert is a common operation method of pumps under offdesign operating conditions. The operating conditions of pumps can be changed effectively by an inverter. Zhang et al. [19] adjusted the flow speed by using a variable speed driver and deduced an energy-saving operating control of pumps based on POET framework. Luo et al. $[20,21]$ constructed a mathematical model based on multi-type pump and pump system with an inverter. They determined the optimal solution to the control strategy of the inverter by combining the golden section and genetic algorithms, and they applied synchronous switch control technique to optimize the adjustment of multiple types of pump systems. The optimal solution was calculated on the basis of the minimum axial power scheduling module. In the present study, based on the ternary theory of fluid mechanics, solid-liquid flow characteristics of a polypropylene axial flow pump in a loop reactor were discussed by combining numerical simulation and experiment. Key attention was paid to the variation laws of pump head and the solid and turbulence energy distributions on the impeller surface under different solid volume fractions. The causes of axial power fluctuation of the pump were discussed, and optimization measures based on head correction were proposed on the basis of the operating control with single invert. Relevant research results could provide references to the optimization of frequency conversion control in a solid-liquid flow pump under off-design operating conditions.

The reminder of this study is organized as follows. Section 3 describes the structure of the axial flow pump in the loop reactor and constructs the hydraulic and test models for the pump. Section 4 introduces a contrast analysis between the external characteristics and internal flows of the model under different media conditions by using the numerical simulation technique. Moreover, the operating control with single invert under off-design operating conditions is proposed. Section 5 summarizes the conclusions.

\section{PHYSICAL MODEL}

\subsection{Model of the axial flow pump in the loop reactor}

Fig. 1 a shows the simplified model of the polypropylene loop reactor. Inlet media of the reactor were liquid propylene and catalyst, whereas the outlet exported the mixture. Pressure of the media, including liquid propylene, catalyst, and polypropylene, is applied by an axial flow pump. The mixture continuously circulated in the reactor until reaction equilibrium was achieved. The reactor had no flow control valve, and the circulation rate of the mixture in the reactor was controlled through inflow and outflow speeds. As only few components were added in the catalysis period, the mixture was simplified into liquid-phase propylene and solid-phase polypropylene. 


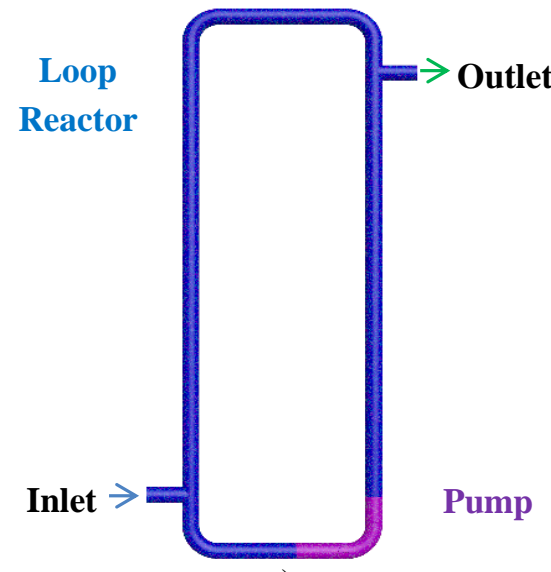

a)

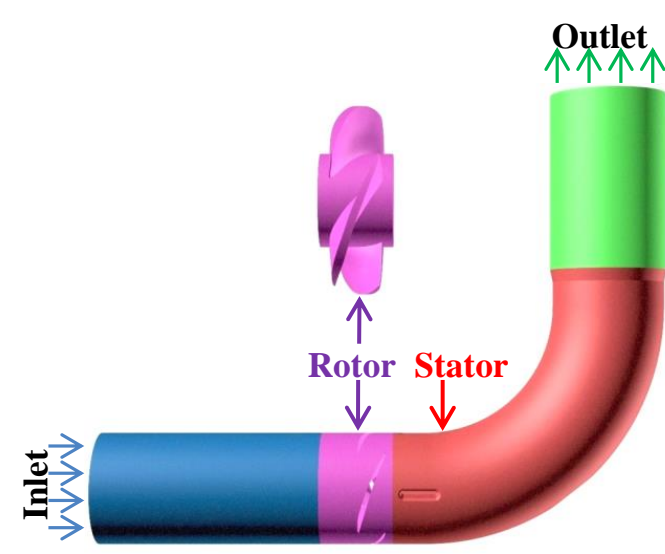

b)

Figure 1: Loop reactor and numerical calculation models of axial flow pump: a) Loop reactor model, b) Numerical calculation model of axial flow pump.

Fig. $1 \mathrm{~b}$ shows the numerical calculation domain of a polypropylene axial flow pump in a loop reactor. The main geometric parameters are as follows: impeller diameter $D_{2}=554.3 \mathrm{~mm}$, hub diameter $D_{h}=274.5 \mathrm{~mm}$, number of blades in an impeller $Z_{i}=4$, and number of guide blades $Z_{b}=4$. The optimal running parameters are as follows: head $H_{\text {des }}=19 \mathrm{~m}$, flow $Q_{\text {des }}=7000 \mathrm{~m}^{3} / \mathrm{h}$ (converted into flow speed $V_{\text {des }}=7.48 \mathrm{~m} / \mathrm{s}$ ), and rotating speed $n=1480 \mathrm{r} / \mathrm{min}$.

\subsection{Calculation methods}

To obtain the internal flow characteristics of an axial flow pump in a loop reactor, the secondorder upwind discrete differential equation was solved using a SIMPLE algorithm based on the ANSYS CFX 16.0 working platform. Convergence precision of iterative computation was $10^{-5}$. The continuously flowing SST $k-\omega$ turbulence model [22], particle model for solid-liquid flows, discrete phase equation model for discrete phase particles, and Gidaspow model for interphase drag force [14] were applied in the numerical calculation. Boundary conditions were set as the total pressure inlet, which hypothesizes the uniform distribution of solid phase, normal speed outlet, wall surface with no liquid slippage and free solid sliding, and scalable wall function. The media used in the numerical calculation of single- and two-phase flows were single-phase clean water, liquid-phase propylene, and solid-phase polypropylene. Table I lists the parameters of the media.

Table I: Main performance parameters.

\begin{tabular}{|c|c|c|c|c|c|c|}
\hline $\boldsymbol{\rho}_{w}\left(\mathrm{~kg} / \mathrm{m}^{3}\right)$ & $\boldsymbol{\mu}_{w}(\mathrm{~Pa} \cdot \mathrm{s})$ & $\boldsymbol{\rho}_{l}\left(\mathrm{~kg} / \mathrm{m}^{3}\right)$ & $\mu_{l}(\mathrm{~Pa} \cdot \mathrm{s})$ & $\boldsymbol{\rho}_{s}\left(\mathrm{~kg} / \mathrm{m}^{3}\right)$ & $\boldsymbol{D}_{p p}(\mathrm{~mm})$ & $\boldsymbol{C}_{\boldsymbol{v}}$ \\
\hline 997.0 & $8.9 \times 10^{-4}$ & 417.7 & $6.0 \times 10^{-5}$ & 900.0 & 2.5 & $5 \% / 10 \% / 15 \%$ \\
\hline
\end{tabular}

In this study, the hexahedral meshing of inlet pipe, impeller, guide vane, and outlet channel was accomplished using ANSYS ICEM 16.0. The value of the boundary layer $y^{+}$of a flow passage component met the requirements of the selected turbulence model. Fig. 2 shows the number of grids and numerically calculated head for independence test. $H_{g 2}$ is the numerically calculated head of meshing scheme Grid 2. The head decreased gradually with the increase in the number of grids. The variation range of the pump head was smaller than $0.2 \%$ when the total number of grids reached 7.15 million. Hence, Grid 2 was selected as an appropriate meshing scheme. Fig. 3 shows the meshing diagram of the impeller. 


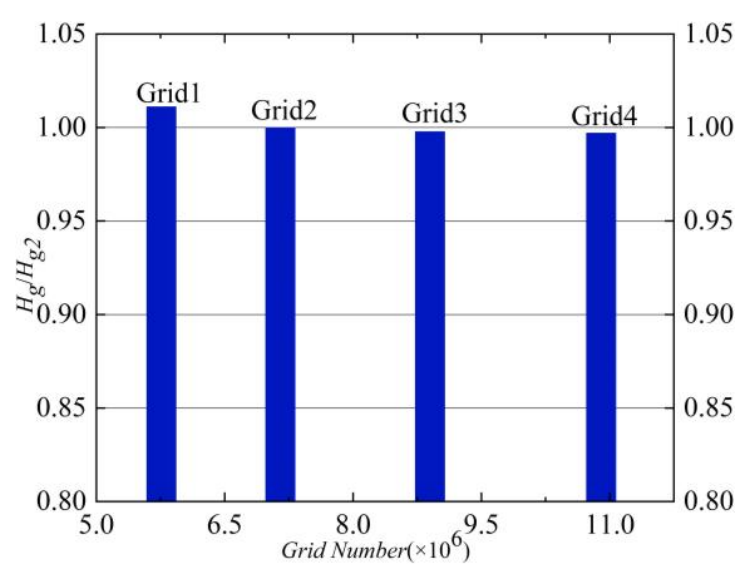

Figure 2: Grid independent examination.

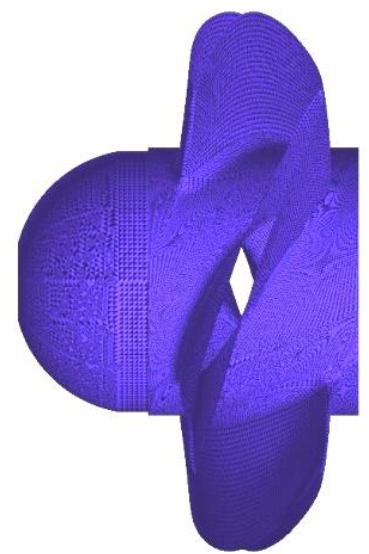

Figure 3: Meshing of the impeller domain under Grid 2.

\subsection{Model test}

As the inlet pressure could reach $4 \mathrm{MPa}$ during practical running of the test object, an assisted pump was added in the pump test device to obtain pressure at inlet. To verify the accuracy of numerical simulation, the test device was built in accordance with the level 1 accuracy of national standards (ISO9906-1999). The measuring equipment has high measurement accuracy. Pressures at the inlet and outlet of the pump were measured via a BOOST.PLD.0201 pressure sensor with a measurement accuracy of $\pm 0.05 \%$. Flow speed was measured using the ENLAI.LDG-DN600 electromagnetic flowmeter with a measurement accuracy of $\pm 0.3 \%$. The axial power of motor was measured by the JNSENSOR.JN-DN rotational torque transducer with a measurement accuracy of $\pm 0.5 \%$. Uncertainty of the entire test device was analysed and was within the allowance range of international standards.

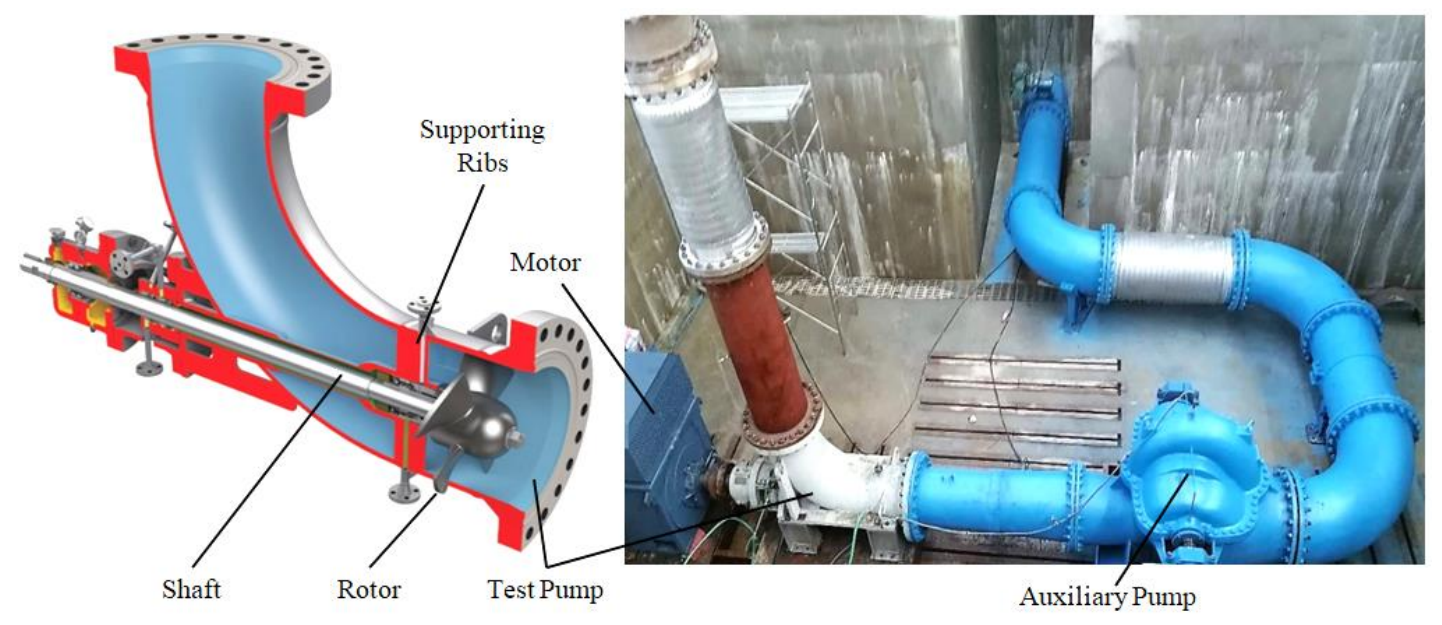

Figure 4: Testing device.

\section{SIMULATION ANALYSIS AND DISCUSSION}

\subsection{Comparison of performances}

Fig. 5 shows the comparison between the numerical calculation under single-phase water of the pump and the test results on hydraulic performance. The variation trend of numerical calculation results agrees with that of test results. Under the design operating conditions, the head is $18.75 \mathrm{~m}$ in numerical calculation, which has an error of $2.6 \%$ with the test head $(19.25 \mathrm{~m})$. The axial power in the numerical calculation is $537.13 \mathrm{~kW}$, which shows $0.1 \%$ difference from the test result $(536.42 \mathrm{~kW})$. The error between the numerical calculation and 
test results under other working conditions is smaller than $5 \%$, thereby meeting the requirements.

Fig. 6 presents the numerical calculation results of the pump head under single-phase propylene and propylene-polypropylene mixture with different solid volume fractions (5\%, $10 \%$, and $15 \%$ ). Variation laws of numerical calculation results under four media conditions are the same. Under the design operating conditions, pump heads under $5 \%, 10 \%$, and $15 \%$ mixing media are $3.6 \%, 8.6 \%$, and $12.9 \%$ lower than that under single-phase propylene, respectively. Moreover, physical properties of the mixing media change with the increase in solid volume fraction. Density of the mixing media can be calculated as:

$$
\rho_{m}=C_{V} \cdot \rho_{s}+\left(1-C_{V}\right) \cdot \rho_{l}
$$

$C_{V}$ is the solid volume fraction, $\rho_{s}$ is the density of polypropylene, and $\rho_{l}$ is the density of propylene. Viscosity of the mixing media can be estimated using Thomas formula:

$$
\mu_{m}=\mu_{l}\left[1+2.5 C_{V}+10.05 C_{V}^{2}+0.00273 \exp \left(16.6 C_{V}\right)\right]
$$

$\mu_{l}$ is the viscosity of propylene. Fig. 6 shows the histogram of relation among mixture density, viscosity, and head under the design operating conditions. The pump head is negatively correlated with media density and viscosity.

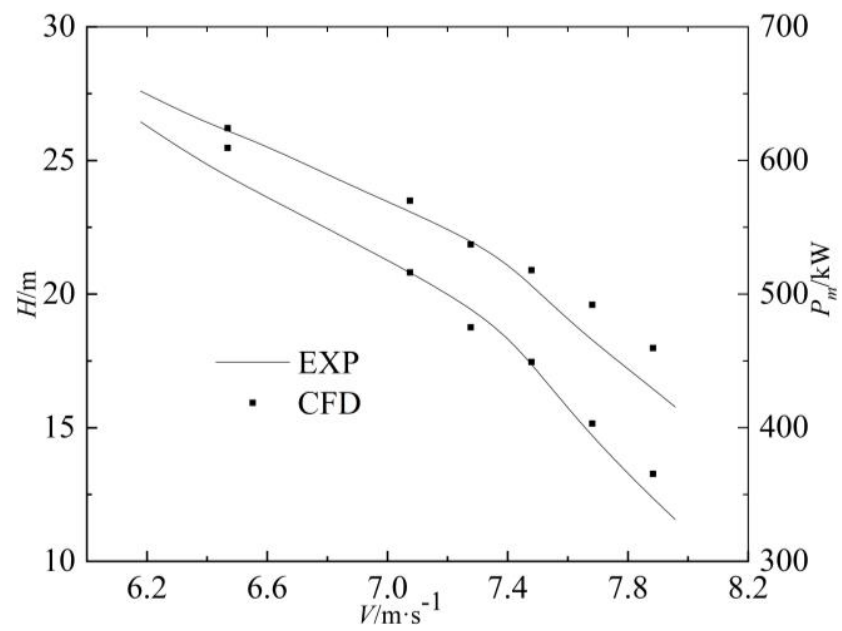

Figure 5: Comparison between numerical calculation and test results under single-phase water.

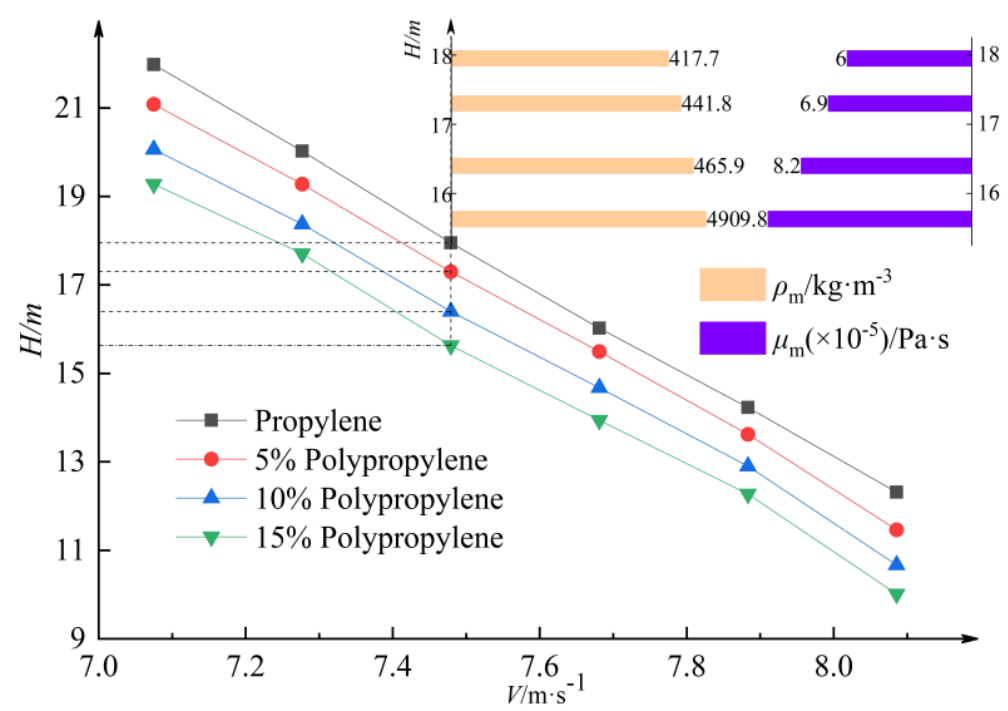

Figure 6: Comparison between numerical calculation and test results under different solid volume fractions. 


\subsection{Analysis of solid volume and turbulence kinetic energy distribution}

Under the design operating conditions, solid volume fractions were set as $5 \%, 10 \%$, and $15 \%$. The diameter of particles was set as $2.5 \mathrm{~mm}$. Fig. 5 shows the solid volume distribution diagrams on pressure and suction surfaces of blades of the mixture and single-phase propylene under different solid volume fractions. Fig. 7 a presents the solid volume distribution diagram on the pressure surface of blades. As the axial feeding solid particles influence the radial rotating blade, few solid particles are in the region and low solid volume fraction exists. Hence, solid particles are mainly distributed at the outlet of blades. Solid volume fraction at the outlet increases with the increase in the setting value of solid volume fraction. However, the solid distribution law on the pressure surface of blades remains under different solid volume fractions, which was approximately $1 / 3$ of the area higher than the setting value.

Fig. $7 \mathrm{~b}$ displays the solid volume distribution diagram on the suction surface of blades. Solid particles are mainly distributed at the outlet of blades, adjacent regions of the hub, and blade-shaped linear arc 1/6 away from the blade inlet. These regions form an L-shaped region. Solid volume fraction on the suction surface of blades decreases sharply compared with that on the suction surface of blades. The solid volume fraction of the L-shaped region is positively related with its setting value. However, the solid distribution law on the suction surface of blades remains under different solid volume fractions, which is approximately $1 / 3$ of the area higher than the setting value.

Fig. 8 a shows the histograms of the calculated results of turbulence kinetic energy under different solid volume fractions (viscosity). Here, $\mu_{p}: \mu_{5}: \mu_{10}: \mu_{15}=1: 1.15: 1.37: 1.63\left(\mu_{p}, \mu_{5}\right.$, $\mu_{10}$, and $\mu_{15}$ are viscosity values) under four solid volume fractions $(0 \%, 5 \%, 10 \%$, and $15 \%)$. On the basis of the formulas of Reynolds number $R e=\rho v d / \mu$, turbulence intensity $l=(0.16 \times R e)^{-1 / 8}$, and turbulence kinetic energy $k=1.5(u l)^{2}, k_{p}: k_{5}: k_{10}: k_{15}=1: 1.04: 1.08: 1.13$, (where $k_{p}, k_{5}, k_{10}$, and $k_{15}$ are turbulence energies) under four solid volume fractions $(0 \%, 5 \%$, $10 \%$, and $15 \%$ ). With the increase of solid volume fraction in the mixing media, the media viscosity, turbulence kinetic energy, and energy dissipation increase, thus the pump head decreases.

Fig. $8 \mathrm{~b}$ presents the distribution cloud map of liquid-phase turbulence kinetic energy on impeller sections of $0.25,0.5$, and 0.75 spans when the solid volume fraction is $15 \%$. Span is defined as the dimensionless distance from the impeller hub to the rims, and it ranges $(0,1)$. An evident region with high turbulence kinetic energy is formed at the impeller outlet. Moreover, the area of this region with high turbulence kinetic energy increases continuously with the increase in span section. Nevertheless, Fig. 8 b shows the liquid-phase turbulence kinetic energy. As the mass fraction of liquid phase changes continuously with the solid volume fraction, the liquid-phase turbulence kinetic energy must be corrected to obtain the value of the suppositional turbulence kinetic energy of the mixture.

Fig. $8 \mathrm{c}$ exhibits the scatter diagram of suppositional turbulence kinetic energy on the impeller under different solid volume fractions. The suppositional turbulence kinetic energy is calculated from the formula $k_{m}=k_{f} / C_{m}=\left(k_{f} \cdot \rho_{m}\right) /\left[\left(1-C_{V}\right) \cdot \rho_{l}\right]$. In Fig. $8 \mathrm{c}$, the red line is the concentrated distribution line when the number of scatter points is higher than $95 \%$. With the increase in solid volume fraction, the suppositional turbulence kinetic energy peak of scatter points increases continuously and reaches the maximum at approximately $0.04 \mathrm{~m}$ away from the impeller outlet. This result agrees with the location of the region with high turbulence kinetic energy in Fig. 6 b. The turbulence kinetic energy is estimated by the area enclosed by the concentrated regional distribution line and the coordination axis. The ratio of suppositional turbulence energies under different solid volume fractions is calculated as $k_{m p}: k_{m 5}: k_{m 10}: k_{m 15}=1: 1.04: 1.08: 1.15$, which is close to the calculated result of the formula. 


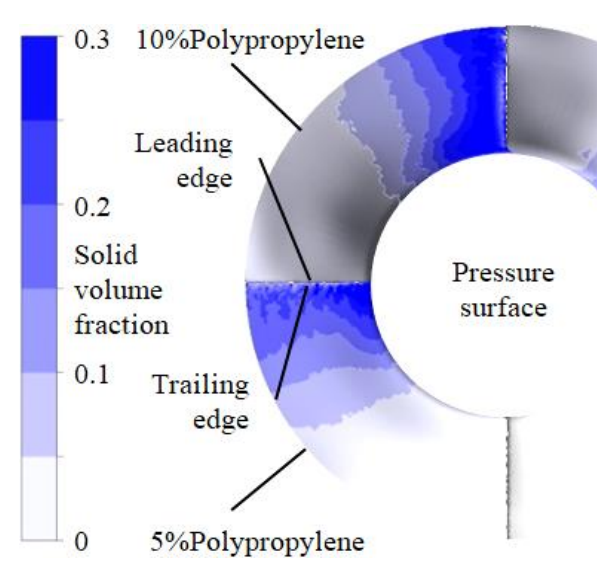

a)

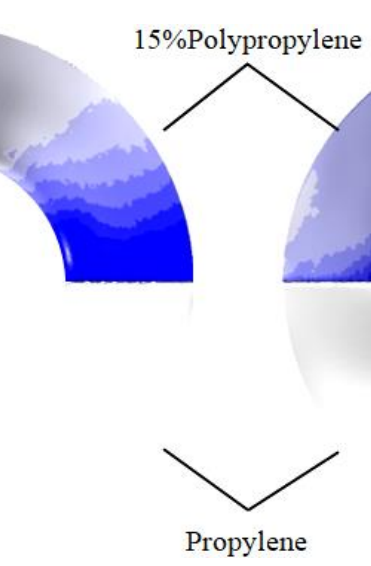

Propylene

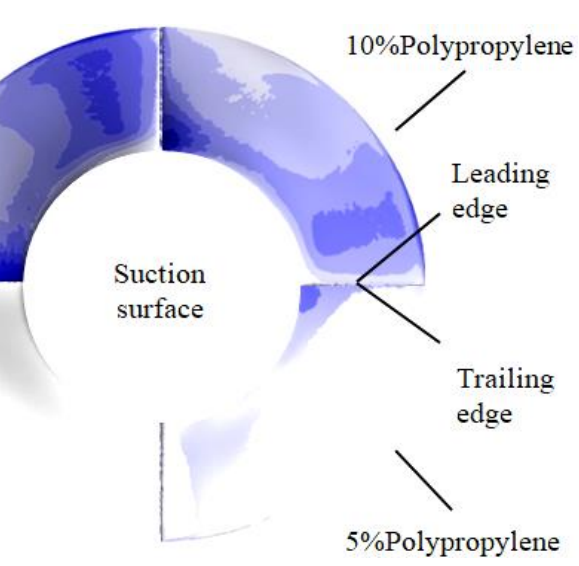

b)

Figure 7: Solid volume distributions on the pressure and suction surfaces of blades: a) pressure surface, b) suction surface.

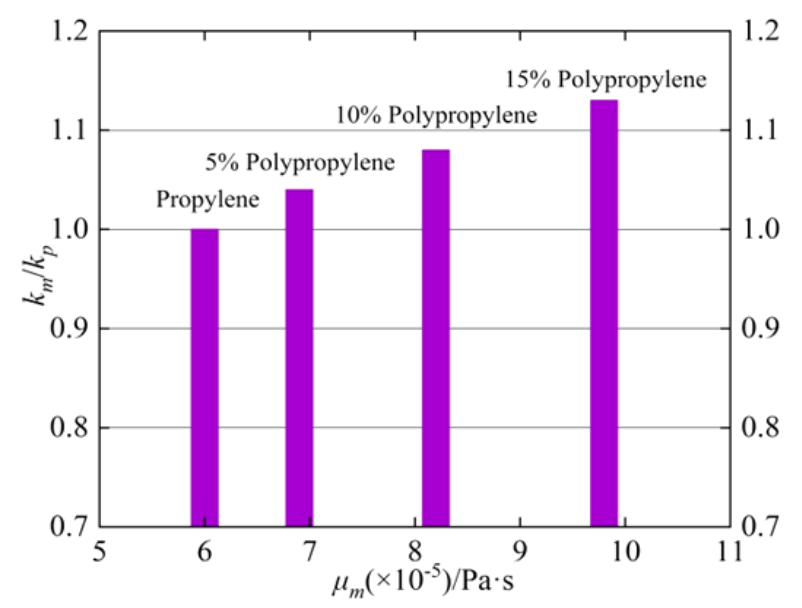

a)

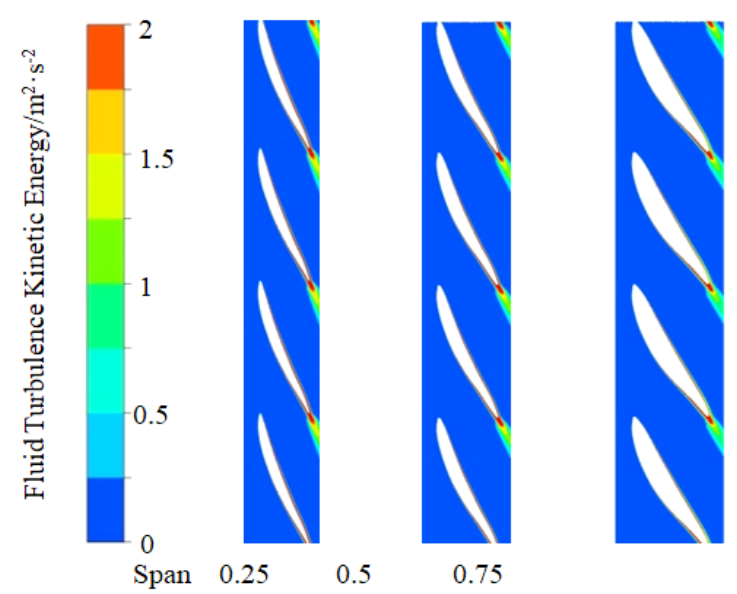

b)

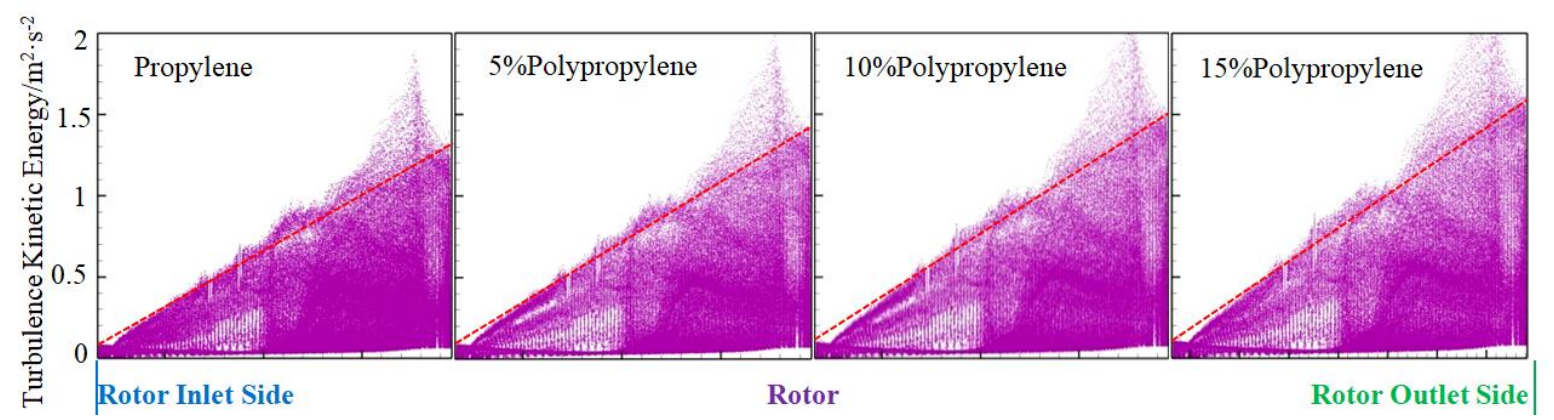

c)

Figure 8: Calculated and numerical calculation results of turbulence kinetic energy: a) Calculated results of turbulence kinetic energy, b) Distribution of turbulence kinetic energy in the flow channel of the impeller, c) Scatter diagram of suppositional turbulence kinetic energy on the impeller.

\subsection{Operating control with single invert}

In practical operation, the head of the axial flow pump meets the requirement that the mixing media complete one circulation in the loop reactor. Thus, the head of the axial flow pump in the loop reactor is higher than or equal to the pipe and bend local head losses:

$$
H_{Z} \geq H_{l}+H_{\xi}=\lambda\left(\operatorname{Re}, \frac{\Delta}{r}\right) \frac{l_{p}}{d} \frac{V^{2}}{2 g}+\left[1.31+0.159\left(\frac{d}{\rho_{r}}\right)^{3.5}\right] \frac{\theta}{90} \frac{V^{2}}{2 g}
$$


$\Delta$ is the absolute roughness of the pipe wall, $l_{p}$ is the length of the circular tube, $d$ is the inner diameter, and $r$ is the radius. $V$ is the flow speed, $\rho_{r}$ is the curvature radius, and $\theta$ is the angle of the bending pipe. Eq. (3) shows that the head loss is positively related, but the pump head of is negatively related with the flow speed. Therefore, a theoretical head $H_{T}$ meets the head loss. In Fig. 9, the actual head of the pump is slightly higher than the theoretical head. Therefore, an energy allowance is available when the mixture enters the pump inlet after one circulation, resulting in operation instabilities, such as axial power fluctuation. To improve the operation stability of the axial flow pump in the loop reactor, the region with energy allowance should be decreased as much as possible. Hence, practical head $H_{Z}$ is controlled within a small range. On the basis of the comparison results in Section 4.1, the head of the axial flow pump in the loop reactor changes with the solid volume fraction, which causes energy allowance.

Fig. 10 exhibits the flow-head relation curves under different solid volume fractions and speeds. Given the same flow speed, the pump head varies with the solid volume fraction. Generally, regulating the openness of the valve is the most effective means of adjusting the off-design operation of the pump [23]. However, the valve is not allowed in the loop reactor and the flow speed of the mixture is controlled through inflow and outflow speeds. The circulation speed in the reactor must meet certain requirements, and it cannot adjust the offdesign operation under different solid volume fractions by regulating flow speeds. In this study, the rotating speed of the pump during practical operation is adjusted by an inverter. Fig. 10 shows that given the setting flow speed, the pump head when the solid volume fraction is $C_{V 2}$ and the rotating speed of the pump reaches $n_{2}$ is equal to that when the solid volume fraction is $C_{V 1}$ and the rotating speed of the pump is $n_{1}$. In this manner, the pump head under different solid volume fractions can be adjusted consistently and effectively without changing the flow speed. As shown in Fig. 9, the actual head of the pump has a certain variation range compared with the theoretical head. In Fig. 10, given the same solid volume fraction, the rotating speed of the pump is adjusted within a certain range to assure that the head is within the rated range.

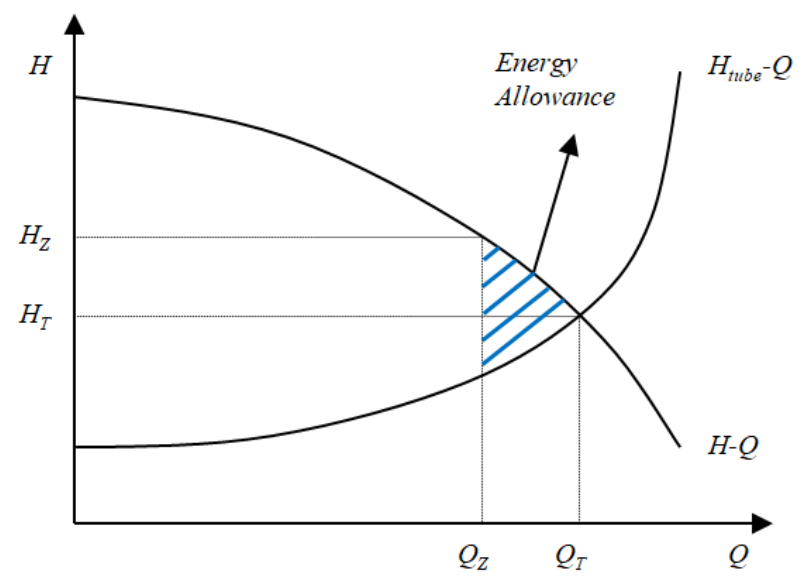

Figure 9: Flow-head relation curves of the pump and pipeline.

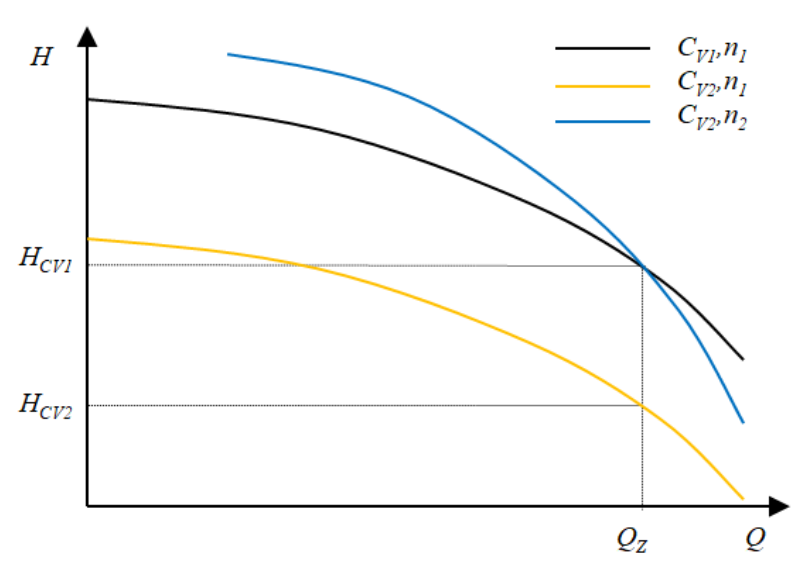

Figure 10: Flow-head relation curves under different solid volume fractions and speeds.

Fig. 11 shows the head correction contour maps of flow and rotating speeds under different solid volume fractions. $Q_{1}, Q_{2}$, and $Q_{3}$ are converted into flow speeds (7.07, 7.27, and $7.47 \mathrm{~m} / \mathrm{s}$, respectively). In the numerical calculation, the pump head $\left(H_{T-0}^{+0.01}\right)$ was in the rated range, and the contour maps of flow speed when the rotating speed ranges between $1460 \mathrm{r} / \mathrm{min}$ and $1530 \mathrm{r} / \mathrm{min}$ are drawn. With the increase in solid volume fraction, the rotating speed increases continuously under a fixed flow speed. The flow speed region within the coverage of the head of $Q_{3}$ expands continuously. For a rated solid volume fraction, the value 
range of the rotating speed of the pump is positively related with the flow speed. The rotating speed in the contour region under different flow speeds $(7.07-7.47 \mathrm{~m} / \mathrm{s})$ is equal to the operating control with single invert when the head is in the rated range under three operating conditions.

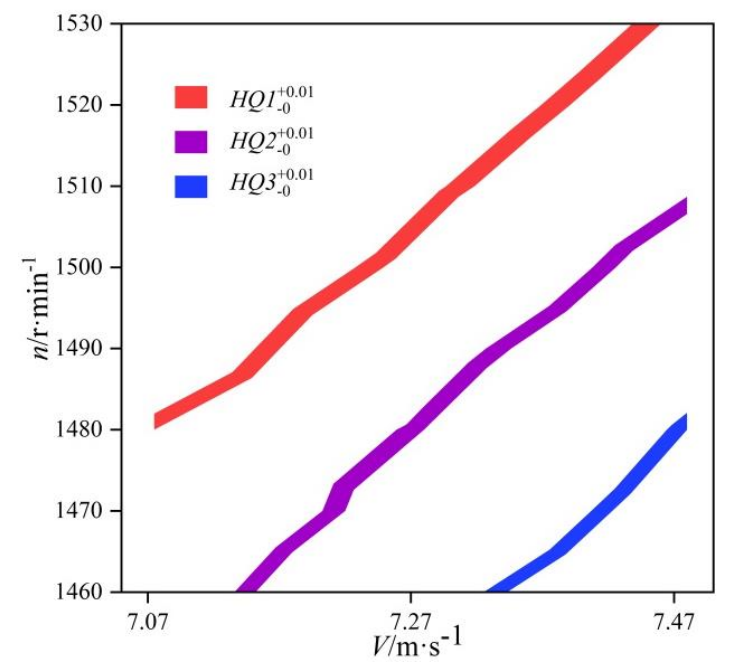

a)

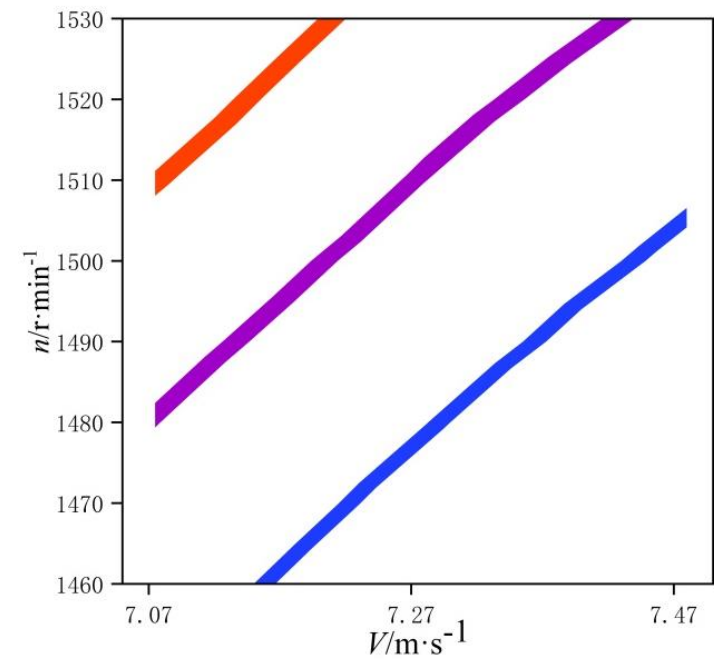

c)

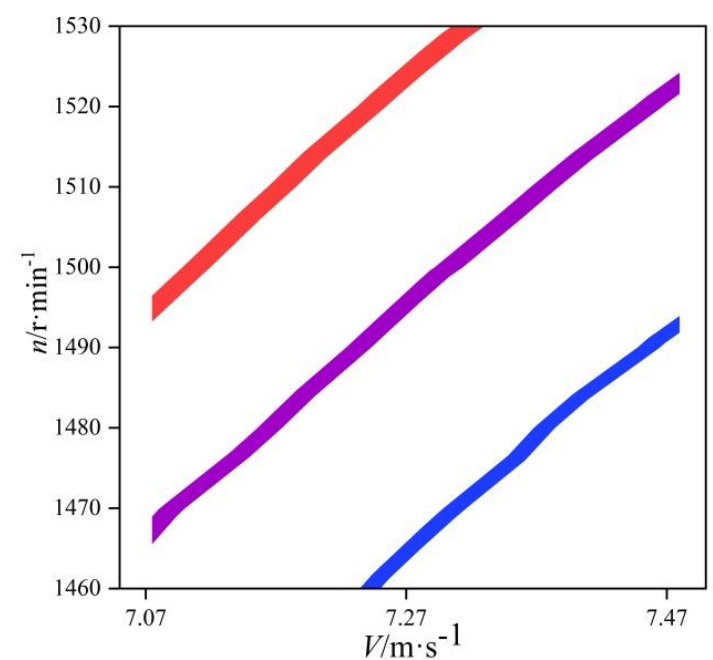

b)

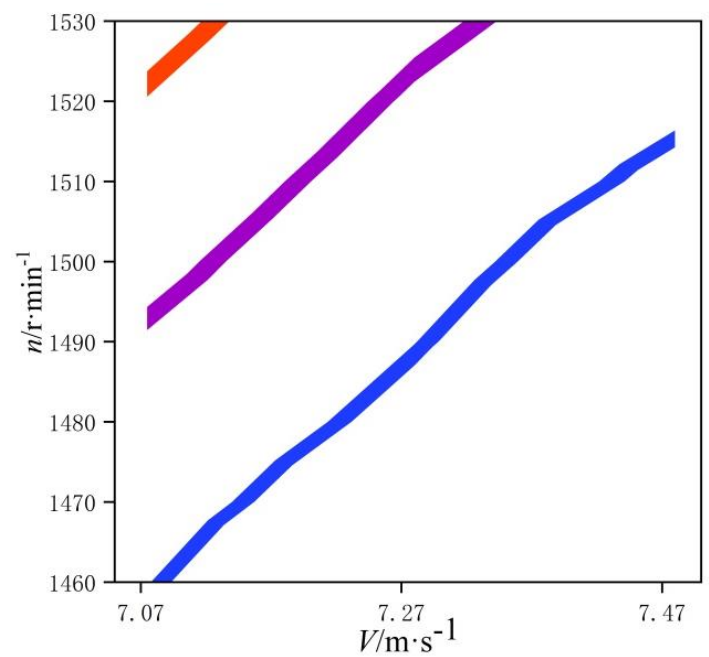

d)

Figure 11: Contour maps of flow and rotating speeds corrected by the head under different solid volume fractions: a) Propylene, b) $5 \%$ Polypropylene, c) $10 \%$ Polypropylene, d) $15 \%$ Polypropylene.

\section{CONCLUSION AND FUTURE WORK}

Based on a physical model, the head of an axial flow pump in a loop reactor and the solidphase and turbulence energy distributions on the blade surface under different solid volume fractions were analysed by combining numerical simulation and experimental study. Analysis results showed that influences of solid-phase concentration on the internal flow state of a pump and the relationship between the solid-phase concentration and axial power fluctuation were disclosed, and corresponding solutions were proposed. Moreover, the correction curve of the pump head under different conditions was obtained on the basis of an operating control with single invert. Several conclusions were drawn:

(1) With the increase in solid volume fraction, the viscosity and turbulence energy of the mixture of liquid-phase propylene and solid-phase polypropylene are increased, thus 
increasing energy loss and decreasing the pump head. The viscosity and turbulence energy of the mixing media are increased by $63 \%$ and $13 \%$, respectively, when the solid volume fraction reaches $15 \%$, whereas the head is decreased by $12.9 \%$. Variation laws of these three parameters are consistent under low concentrations.

(2) The pump head may change under different solid volume fractions of the mixing media, resulting in energy allowance in the loop reactor. This case is one of main causes of the axial power fluctuation of the axial flow pump in the loop reactor.

(3) Operating control with single invert can effectively regulate the off-design operation of the axial flow pump in the loop reactor under different states of media. The region of stable operation of the axial flow pump is selected on the basis of three groups of numerical calculation results of the rated head. Different head correction operating controls are proposed under different solid volume fractions and flow speeds. The optimal correction curves of the rotating speed of the pump are obtained.

In this study, the relationship among the viscosity, turbulence energy of mixing media, and pump head are determined by combining experimental study and numerical calculation. The results provide certain references for investigating the influences of media conditions on pump performance. Measured data of the viscosity of mixture with different solid-phase concentrations are lacking. Thus, numerical values of the viscosity of solid-liquid mixing media should be corrected in future studies to obtain a further accurate relation between the viscosity and pump head.

\section{REFERENCES}

[1] Zhao, B.; Yuan, S.; Liu, H.; Huang, Z.; Tan, M. (2008). Simulation of solid-liquid two-phase turbulent flow in double-channel pump based on Mixture model, Transactions of the Chinese Society of Agricultural Engineering, Vol. 25, No. 1, 7-12, doi:10.3969/j.issn.10026819.2008.1.002

[2] Li, Y.-J.; Wang, F.-J. (2007). Numerical investigation of performance of an axial-flow pump with inducer, Journal of Hydrodynamics, Vol. 19, No. 6, 705-711, doi:10.1016/S1001-6058(08)60007$\underline{4}$

[3] Melo, P. A.; Pinto, J. C.; Biscaia, E. C. (2001). Characterization of the residence time distribution in loop reactors, Chemical Engineering Science, Vol. 56, No. 8, 2703-2713, doi:10.1016/S00092509(00)00517-0

[4] Gertzos, K. P.; Nikolakopoulos, P. G.; Papadopoulos, C. A. (2008). CFD analysis of journal bearing hydrodynamic lubrication by Bingham lubricant, Tribology International, Vol. 41, No. 12, 1190-1204, doi:10.1016/j.triboint.2008.03.002

[5] Durst, F.; Miloievic, D.; Schönung, B. (1984). Eulerian and Lagrangian predictions of particulate two-phase flows: a numerical study, Applied Mathematical Modelling, Vol. 8, No. 2, 101-115, doi: $10.1016 / 0307-904 \times(84) 90062-3$

[6] Pfleger, D.; Gomes, S.; Gilbert, N.; Wagner, H.-G. (1999). Hydrodynamic simulations of laboratory scale bubble columns fundamental studies of the Eulerian-Eulerian modelling approach, Chemical Engineering Science, Vol. 54, No. 21, 5091-5099, doi:10.1016/S00092509(99)00261-4

[7] Kalteh, M.; Abbassi, A.; Saffar-Avval, M.; Harting, J. (2011). Eulerian-Eulerian two-phase numerical simulation of nanofluid laminar forced convection in a microchannel, International Journal of Heat and Fluid Flow, Vol. 32, No. 1, 107-116, doi:10.1016/ j.ijheatfluidflow.2010.08.001

[8] Lee, B. E.; Tu, J. Y.; Fletcher, C. A. J. (2002). On numerical modeling of particle-wall impaction in relation to erosion prediction: Eulerian versus Lagrangian method, Wear, Vol. 252, No. 3-4, 179-188, doi:10.1016/S0043-1648(01)00838-9

[9] Van Sint Annaland, M.; Bokkers, G. A.; Goldschmidt, M. J. V.; Olaofe O. O.; van der Hoef, M. A.; Kuipers, J. A. M. (2009). Development of a multi-fluid model for poly-disperse dense gas-solid 
fluidised beds, Part II: Segregation in binary particle mixtures, Chemical Engineering Science, Vol. 64, No. 20, 4237-4246, doi:10.1016/j.ces.2009.06.043

[10] Solenthaler, B.; Schlafli, J.; Pajarola, R. (2010). A unified particle model for fluid-solid interactions, Computer Animation and Virtual Worlds, Vol. 18, No. 1, 69-82, doi:10.1002/cav.162

[11] Shi, B.; Wei, J. (2014). Numerical simulation of 3D solid-liquid turbulent flow in a low specific speed centrifugal pump: flow field analysis, Advances in Mechanical Engineering, Vol. 6, Paper 814108, 11 pages, doi:10.1155/2014/814108

[12] Shi, B.; Wei, J.; Zhang, Y. (2017). A novel experimental facility for measuring internal flow of solid-liquid two-phase flow in a centrifugal pump by PIV, International Journal of Multiphase Flow, Vol. 18, 266-276, doi:10.1016/j.ijmultiphaseflow.2016.11.002

[13] Zhang, Y.; Li, Y.; Zhu, Z.; Cui, B. (2014). Computational analysis of centrifugal pump delivering solid-liquid two-phase flow during startup period, Chinese Journal of Mechanical Engineering, Vol. 27, No. 1, 178-185, doi:10.3901/CJME.2014.01.178

[14] Shi, W.; Xing, J.; Zhang, D.; Chen, K.; Cheng, C. (2014). Numerical simulation and optimization of solid-liquid two-phase turbulent flow in back swept axial pump, Transactions of the Chinese Society of Agricultural Engineering, Vol. 30, No. 11, 76-82, doi:10.3969/j.issn.10026819.2014.11.010

[15] Lei, H. M.; Xiao, Y. X.; Chen, F. N.; Ahn, S. H.; Wang, Z. W.; Gui, Z. H.; Luo, Y. Y.; Zhao, X. R. (2018). Numerical simulation of solid-liquid two-phase flow in a centrifugal pump with different wear blades degree, IOP Conference Series: Earth and Environmental Science, Vol. 163, No. 1, Paper 012027, 11 pages, doi:10.1088/1755-1315/163/1/012027

[16] Shi, D.-P.; Luo, Z.-H.; Zheng, Z.-W. (2010). Numerical simulation of liquid-solid two-phase flow in a tubular loop polymerization reactor, Powder Technology, Vol. 198, No. 1, 135-143, doi:10.1016/j.powtec.2009.11.002

[17] Yan, W.-C.; Shi, D.-P.; Luo, Z.-H.; Lu, Y.-H. (2011). Three-dimensional CFD study of liquidsolid flow behaviors in tubular loop polymerization reactors: the effect of guide vane, Chemical Engineering Science, Vol. 66, No. 18, 4127-4137, doi:10.1016/j.ces.2011.05.039

[18] Li, Y.; Ma, Y.; Reddy, R. K.; Vijay, S.; Elovanio, E.; Wurnitsch, C.; Nandakumar, K. (2015). CFD investigations of particle segregation and dispersion mechanisms inside a polyolefin 8-leg loop reactor of industrial scale, Powder Technology, Vol. 284, 95-111, doi:10.1016/ j.powtec.2015.06.031

[19] Zhang, H.; Xia, X.; Zhang, J. (2012). Optimal sizing and operation of pumping systems to achieve energy efficiency and load shifting, Electric Power Systems Research, Vol. 86, 41-50, doi:10.1016/j.epsr.2011.12.002

[20] Luo, Y.; Yuan, S.; Sun, H.; Guo, Y. (2015). Energy-saving control model of inverter for centrifugal pump systems, Advances in Mechanical Engineering, Vol. 7, No. 7, Paper 1687814015589491, 12 pages, doi:10.1177/1687814015589491

[21] Luo, Y.; Xiong, Z.; Sun, H.; Guo, Y. (2017). Research on energy-saving operation control model of the multi-type configuration centrifugal pump system with single invert, Advances in Mechanical Engineering, Vol. 9, No. 7, Paper 1687814017707650, 10 pages, doi: $\underline{10.1177 / 1687814017707650}$

[22] Yang, J.; Pavesi, G.; Liu, X.; Xie, T.; Liu, J. (2018). Unsteady flow characteristics regarding hump instability in the first stage of a multistage pump-turbine in pump mode, Renewable Energy, Vol. 127, 377-385, doi:10.1016/j.renene.2018.04.069

[23] Li, X.; Jiang, Z.; Zhu, Z.; Si, Q.; Li, Y. (2018). Entropy generation analysis for the cavitating head-drop characteristic of a centrifugal pump, Proceedings of the Institution of Mechanical Engineers Part C, Journal of Mechanical Engineering Science, Vol. 232, No. 24, 4637-4646, doi: $\underline{10.1177 / 0954406217753458}$ 